\title{
Development of a Computer Based Problem-Oriented Automated Remote Leakage Detection Control System
}

\author{
Alisa Kosach $^{1, *}$, and Evgeny Kovshov ${ }^{1}$ \\ ${ }^{1}$ JSC «NIKIMT-Atomstroy», Moscow, Russian Federation
}

\begin{abstract}
We explore the architecture and the concept of building an automation remote leakage detection system, in particular - a system for collection and processing of information. As practice shows, the development of those systems is a lengthy and time-consuming process involving management of heterogeneous hardware and analysis of large amounts of data. To implement the task, it is proposed to use the SCADA industry standard, universal communication protocols, and for data processing - open source database management systems and cluster analysis algorithms. A universal approach makes it possible to develop a scalable problem-oriented system. This approach takes into account the information received and processed during the leakage detection process.
\end{abstract}

\section{Introduction}

Leakage detection is a type of nondestructive testing performed to identify crosscutting defects. It is based on registration of passing of probe material through the defects. During the leakage detection process it is necessary to ensure safety of the staff, high sensitivity of the control process and integration of the control systems directly into the process line. Commonly instrumental leakage detection methods are used, they require complex equipment management and working with semi-structured data. Such remote leakage detection systems are unique, created especially to solve specific problems, and there is no single universal approach for their automation.

Today, there is a large number of tools and process automation systems, including the ones for nondestructive testing. The lack of a universal approach, diversity and often incompatibility of the equipment used, analysis of large amounts of unstructured data turn the development of an automated control system into a long and laborious task. As a result the systems of this kind often can be described as containing poorly aligned components, inefficient data processing algorithms and complex at maintaining performance. In addition, the requirements of a modern enterprise demand using of a single information space, so the measurement equipment and the remote leakage detection systems must be selected carefully according to the characteristics allowing being included in this space.

The target of the submitted work is to develop an automated computer based remote leakage detection control system.

To achieve this target the following research objectives should be addressed:
- development of the control system's architecture and the choice of the equipment for introduction it into a single information space;

- development of the data analysis system for processing the data obtained during the control process;

- development of a unified approach to the automation of remote leakage detection, including the one based on OPC servers and set of Modbus protocols.

\section{Data Analysis Unit}

Evaluation of the product is based on a comparison of the values of the flow test substance, penetrating through the defects, with the acceptance level, depending on the norm of tightness (Fig. 1). If the acceptance level is exceeded the product is considered to be leaking. Before the evaluation process it is necessary to "clean" the signal of noise and possible artefacts - distorted values resulting from the measurement and data transmission errors.

Also, any system has signal fluctuations that might be flattened using averaging. Such filters are used in processing of images to make changes of their sharpness [1]. In this case, each point on the graph is replaced by a weighted average of its neighborhood that provides smoothing of the signal. Filter kernel (i.e. each weight value when calculating the average) can be varied depending on the desired result.

Noise compensation can be performed in a static or a dynamic mode. The static mode involves subtraction of the noise part from the signal, the noise part is a constant value obtained when configuring the system. The dynamic compensation is based on monitoring of the slope angle of the signal curve and subtracting a straight line with a slope coefficient $\operatorname{tg} \alpha$. 


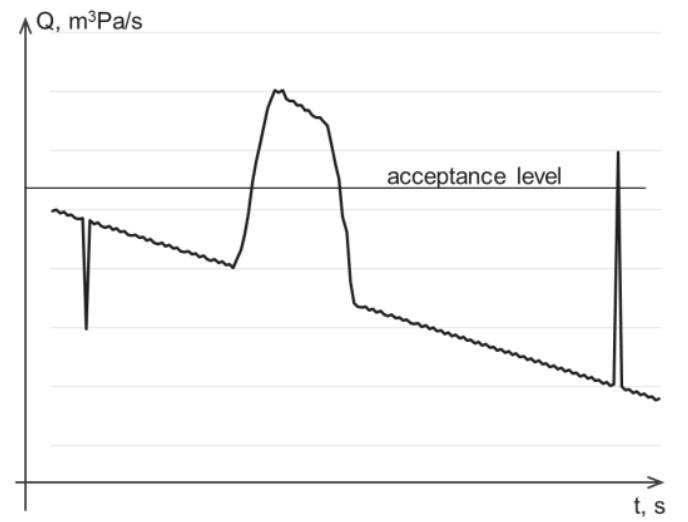

Fig. 1. Graph of the flow the test substance over time.

Removal of artifacts and the further search for the maximum value is based on the method of cluster analysis for pairwise weighted average [2]. As the criterion for connecting into clusters Euclidean distance is applied.

$$
\rho_{E}(x ; y)=\sqrt{\left(Q_{x}-Q_{y}\right)^{2}+\left(t_{x}-t_{y}\right)^{2}}
$$

where $\rho_{\mathrm{E}}(\mathrm{x} ; \mathrm{y})$ is the distance between the values of $\mathrm{x}$ and $\mathrm{y}$; $\mathrm{Q}_{\mathrm{x}}, \mathrm{Q}_{\mathrm{y}}$ are the corresponding coordinates along the $\mathrm{Y}$ axis; $\mathrm{t}_{\mathrm{x}}, \mathrm{t}_{\mathrm{y}}$ are the corresponding coordinates along the horizontal axis.

The algorithm distributing different values into clusters is used, in which the values are combined into groups based on general criteria. Gradually the scatter of the criteria in each cluster increases as the clusters themselves do. Because the curves representing the time dependency of the flow can be characterized as smoothly changing it can be assumed that the artifacts will appear in the clusters with a minimum number of members. To remove the corrupted values it is enough to remove the most numerically small clusters.

By using the method of cluster analysis the amount of the resources spent on the search for the maximum value is reduced. It uses the linear search algorithm, in which the first is the search for the cluster with a maximum average value of its members. After that, the maximum value is found in that cluster. When using this algorithm, one can be sure that the excess of the acceptance level is not caused by any noise fluctuations or random artifacts.

The possibility of modeling and documentation of data in an off-line mode is provided. This mode includes: creating a graph representing the time dependency of the flow of the test substance, data re-filtering and evaluation of the product shelf-life. According to the results of the monitoring process technological documents can be created in electronic format and printed on paper (protocols and reports) on demand.

\section{Practical usage}

This concept is applied to create the computer based automated system for remote mass-spectrometry leakage detection in canisters' closing welds after filling them with radioactive waste.

OPC servers and SCADA technology have been used to solve the problem. The SCADA technology [3] (Supervisory Control And Data Acquisition) is a hardware and software system designed for managing production processes in real time. The software that implements this technology allows communicating with the automated objects via the input-output drivers or servers. Thus, systems with any number of equipment included in the in-line production line can be simultaneously controlled.

OPC is a technology that uses the single interface to control devices [4]. Its use is especially important for remote leakage detection system. Usually, software developers have to include in the system the set of readymade drivers and use different tools for developing original software for interacting with non-standard lowlevel devices. When replacing a device with the similar one made by another manufacturer it is necessary to change a part of the software for its management. OPC unified interface allows different software modules, manufactured by different companies successfully communicate with each other.

The control system for remote leakage detection is based on a distributed architecture (Fig. 2). This architecture consists of several control centers which are connected to a common control circuit. Because of this the distributed management architecture has no restrictions in the performance of the control center and the length of the communication channels. In addition, it is characterized by wide possibilities of modernization, changing and increasing capacity, component approach, easy commissioning and maintenance. The main software that implements the algorithm is located in the operator's computer, which receives data (with OPCserver and Modbus protocols) from the controller and the leak detector connected with the hub. The controller communicates with the digital and analog signals obtained from pressure sensors, valves, roughing pumps etc.

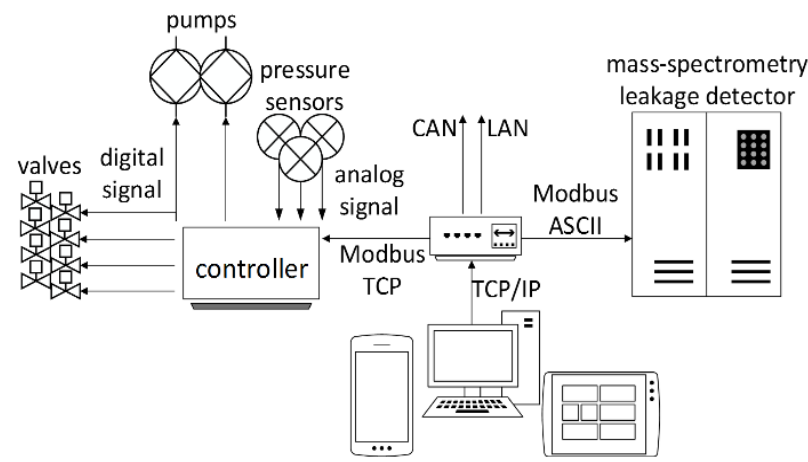

Fig. 2. The control circuit of the computer based automated remote leakage detection system.

For development of the applied software for the remote leakage detection system the open source and freeware public library Pascal SCADA and Lazarus IDE in Object Pascal for the compiler Free Pascal have been used. These software tools are cross-platform, they refer 
to the concept of RAD (Rapid Application Development) and they are not proprietary, thereby constantly improving. For information exchange between the parts of the system the OPC-servers (OPC UA, OPC DA) and the data exchange protocols Modbus RTU and Modbus TCP are used. Communication is performed in CSV format [5] and PostgreSQL open source software is used as a relational DBMS (database management system) [6]. Choosing the DBMS PostgreSQL is caused by its compact data representation, a clear hierarchy of levels that determine the degree of data detail, low probability of obtaining errors and data inconsistencies.

\section{Conclusion}

During this work the hardware and software architecture of the control computer based remote leakage detection system, including the tools for integration of the equipment into a common information space, were defined and built, also the concept of the system analyzing data obtained as a result of a control process has been developed, and a unified approach to automation of remote leakage detection, (also taking into account the OPC-servers and Modbus protocols) has been designed.

With the SCADA and OPC technologies controlling of a system is implemented regardless of different communication protocols between physical devices and can be integrated into the technological line of the enterprise.

\section{References}

1. N.N. Mitropolsky, E.E. Kovshov, N.N. Hue, N.C. Minh, Control Systems and Information Technologies, 37, 80 (2009)

2. P.A Jaskowiak., D. Moulavi, A.C.S. Furtado, Knowledge and Information Systems, 47, 329 (2016)

3. C.P. Sandip, S. Pritimoy, Information Management \& Computer Security Journal, 16, 398 (2008)

4. A.A. Karataev, V.P. Palshin, Z.M. Yarmukhamedova, Herald of KazNTU, 3, 138 (2014)

5. CSV Files. URL: http://www.csvreader.com

6. E.E. Kovshov, A.E. Volkov, G.G. Charaev, Vestnik MSTU STANKIN, 27, 136 (2013) 